\title{
Minimum Delay Packet-sizing for Linear Multi-hop Networks with Cooperative Transmissions
}

\author{
Ning Wen and Randall A. Berry \\ Department of Electrical Engineering and Computer Science \\ Northwestern University, Evanston, Illinois 60208 \\ Email: \{nwen,rberry\}@eecs.northwestern.edu
}

\begin{abstract}
We consider optimizing packet sizes and the reuse factor to minimize the delay required to send a message between two nodes in a linear multi-hop wireless networks subject to a reliability constraint. In earlier work, this problem was considered for a network in which each node only decoded the transmission of the previous node, treating the transmissions of all other nodes as noise. Here, we consider a cooperative transmission scheme in which a node uses the transmissions of all previous nodes to decode a given message. We analyze the growth of the delay as well as the optimized system parameters as a function of the message size.
\end{abstract}

\section{INTRODUCTION}

In a store-and-forward wire-line network with error free links, ignoring any overhead per packet, the end-to-end delay to send a message over multiple hops is minimized by dividing the message into as small of packets as possible so as to benefit from pipelining. If overhead is not ignored, there is an optimal packet-size that balances this pipelining effect with the amortization of overhead given by using larger packets. In multi-hop wireless networks, the picture becomes more complicated due to the interference between different "links," the presence of half-duplex constraints and that links are not well modeled as error-free. To address these issues in [1] a model was studied for optimizing both the packet-size and reuse factor in a linear multi-hop wireless network with regularly spaced nodes. These parameters where optimized to minimize the end-to-end delay for sending a fixed amount of data with a given end-to-end reliability constraint, modeled using error exponents. Related models have also been studied in [2]-[5].

In [1], it is assumed that each node decodes and relays each message based only on the received signal from its nearest neighbor, treating other simultaneous transmissions as noise. In particular, a node ignores transmissions by nodes other than its nearest neighbor. It is well-known that the throughput of such a multi-hop network can be improved by using various cooperative schemes, e.g. [6], [7]. Here we consider a simple cooperative reception scheme as in [7], in which each node uses the transmissions of multiple previous nodes to decode a given message. Given such a scheme, we again consider optimizing the packet sizes and re-use factor. As in [1], we focus on the asymptotic growth in the total delay as the message size increases. In this regime, we show that the

This work was supported in part by the DARPA IT-MANET program under grant W911NF-07-1-0028 and by NSF under grant CCF-0905224. optimal number of packets scales in the same way as without cooperative receptions, but the optimal re-use factor increases without bound as the signal-to-noise ratio (SNR) increases, while without cooperative reception this approaches a bounded constant. Numerical results comparing this scheme to that without cooperative reception are also given.

\section{SySTEM MOdeL}

As in [1], we consider an infinite one-dimensional model, where all nodes are regularly spaced on a line and we normalize the distance between nodes to be 1 . One node $x$ is assumed to have $L$ nats $^{1}$ of data to send to another node $y$. Let $H-1$ be the number of nodes between $x$ and $y$, each of which is assumed to be a relay for the message (i.e. the number of "hops" is $H$ ). To simplify the analysis, we assume the queuing delay on each hop is zero. This is reasonable assuming that the given flow has higher priority over other flows in the network. All nodes are assumed to transmit in the same frequency band (with normalized bandwidth of 1) and the interference is treated as Gaussian noise. The channel between any pair of nodes is modeled as distance dependent path-loss with additive Gaussian noise. All nodes transmit with power $P$ and the noise power is $N_{0}$. We assume the nodes employ a regular TDM-schedule with reuse factor $K$, so that in time-slot $t$ nodes $n K+(t \bmod K)$ are allowed to transmit, for $n=\ldots,-1,0,1, \ldots$. Furthermore, we assume that all nodes (including those not between the source and destination) always transmit in their assigned time-slot, so that there are no "edge effects" in terms of the received interference.

Next we describe the cooperative reception scheme we consider, which we refer to as using "redundant receptions." Each packet will be encoded into a codeword and transmitted by each node following the TDM schedule. For a given re-use factor $K$, each node stores the transmissions of a message from its $K-1$ preceding neighbors and uses these for decoding (see Fig. 1), treating all other interference as noise. The distance between the transmitters and the receiver for these $K-1$ transmissions are $K-1, K-2, \ldots, 1$, respectively, and so there will be receptions with $K-1$ different signal-to-noise-

\footnotetext{
${ }^{1}$ To simplify notation, we use nat as the unit of information.
} 
plus-interference ratios (SINRs). ${ }^{2}$
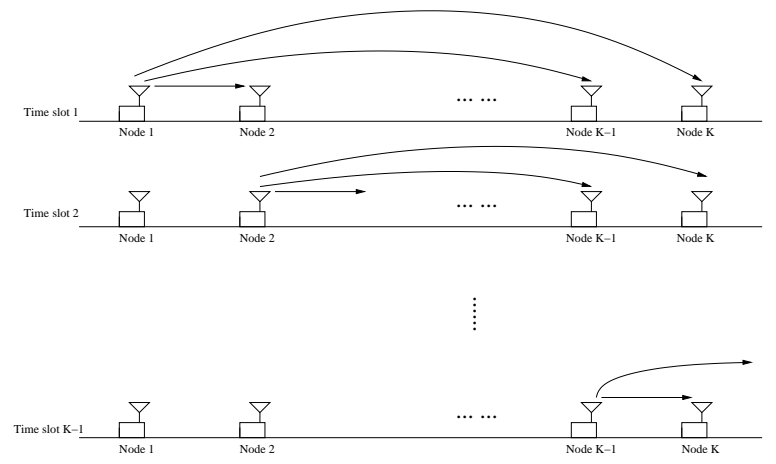

Fig. 1. Example of using redundant receptions.

$$
\begin{aligned}
& \text { Let } \\
& \gamma_{j}\left(\frac{N_{0}}{P}, K\right)=\frac{j^{-\alpha}}{\frac{N_{0}}{P}+\sum_{i=1}^{\infty}\left[(i K+j)^{-\alpha}+(i K-j)^{-\alpha}\right]} .
\end{aligned}
$$

denote the received SINR for a packet transmitted by the node $j$ hops away from a given receiver, where $\alpha$ is the path-loss exponent. Let $\gamma$ be the vector of the $K-1$ SINRs used for decoding. Given that a packet with block-length $N_{h b}$ containing $L_{b}$ nats of information is successfully received by all of the $K$ preceding neighbors of a given node $i$, the probability, $P_{h b}$ that the packet is not received correctly by node $i$ can be bounded as [8]:

$$
P_{h b} \leq \exp \left(\rho L_{b}-N_{h b}\left(\tilde{E}_{0}(\rho, \gamma)\right)\right),
$$

for any $\rho \in[0,1]$, where

$$
\tilde{E}_{0}(\rho, \gamma) \rho \sum_{i=1}^{K-1} \log \left(1+\frac{\gamma_{i}\left(\frac{N_{0}}{P}, K\right)}{1+\rho}\right) .
$$

This follows from viewing the $K-1$ received transmissions as the outputs of $K-1$ parallel Gaussian channels with different SINRs and bounding the error exponent for these channels as in [8] ${ }^{3}$. To apply this result we require that each node re-encodes a packet using a different codeword on each hop.

Given an upper bound $\eta_{h b}$ on the block error probability $P_{h b}$ for a single hop, the minimum $N_{h b}$ satisfying

$$
N_{h b} \rho \sum_{i=1}^{K-1} \log \left(1+\frac{\gamma_{i}\left(\frac{N_{0}}{P}, K\right)}{1+\rho}\right) \geq \rho L_{b}-\log \eta_{h b}
$$

is the minimum delay for sending a block of $L_{b}$ nats over that hop for which we can use (3) to guarantee that the reliability constraint is met. In the rest of the paper, we use this value of $N_{h b}$ as the minimum delay for each hop.

${ }^{2}$ If the first node of the $H$ hop propagation interval is the source node, then nodes $2,3, \ldots, K-1$ receive fewer than $K-1$ copies of the message. To simplify the discussion, we assume that the source node transmits in each time slot with different power to mimic the transmissions from the nodes ahead of it, and all the other nodes receive the same number of duplicate copies.

${ }^{3}$ In the usual parallel channel model the transmissions are sent simultaneously by a single transmitter, while here they are sent at different times by different transmitters. Conditioned on all preceding transmitters receiving the packet, this difference does not matter.
We consider a model in which the source divides the $L$ nats of traffic into $m$ equal-sized packets containing $L_{b}=\frac{L}{m}+h$ bits, where $h$ denotes an extra overhead needed per packet. The objective is to minimize the total delay given a reliability constraint that specifies the end-to-end probability of error for the entire message must be at least $\eta$.

\section{ASYMPTOTIC DELAY ANALYSiS}

\section{A. Hop-by-hop Decoding Scheme}

We begin by considering a hop-by-hop coding scheme, in which packets are coded individually for each hop. In this case, to guarantee an end-to-end reliability of $\eta$, it is sufficient to guarantee a hop-by-hop reliability of $\eta_{h b}=\frac{\eta}{m H}$.

It follows from (4) that the delay $D_{1}$ of sending one packet over one hop must now satisfy

$$
D_{1} \geq \frac{\left(\frac{L}{m}+h\right)-\frac{1}{\rho} \log \frac{\eta}{m H}}{\sum_{j=1}^{K-1} \log \left(1+\frac{\gamma_{j}\left(\frac{N_{0}}{P}, K\right)}{1+\rho}\right)} .
$$

The number of time slots from the source node sending out the first block until the destination node receives the last block is $H+(m-1) K$. Thus, the total delay should satisfy

$$
D \geq \frac{(H+(m-1) K)\left[\left(\frac{L}{m}+h\right)-\frac{1}{\rho} \log \frac{\eta}{m H}\right]}{\sum_{j=1}^{K-1} \log \left(1+\frac{\gamma_{j}\left(\frac{N_{0}}{P}, K\right)}{1+\rho}\right)} .
$$

We consider the behavior of the bound in (6) when the length of the message $L$ goes to $\infty$. First, we rewrite the right-hand side of (6) as

$$
\begin{gathered}
D=\frac{K}{\sum_{j=1}^{K-1} \log \left(1+\frac{\gamma_{j}\left(\frac{N_{0}}{P}, K\right)}{1+\rho}\right)}\left(L+\frac{1}{\rho} m \log m\right. \\
\left.+b_{1} \frac{L}{m K}+\left(h+b_{2}\right) m+\frac{b_{1} \log m}{K \rho}+\frac{1}{K} b_{1}\left(h+b_{2}\right)\right),
\end{gathered}
$$

where $b_{1}=H-K$, and $b_{2}=-\log \frac{\eta}{H}$. This expression is identical to the corresponding one in [1] expect for the coefficient $\frac{K}{\sum_{j=1}^{K-1} \log \left(1+\frac{\gamma_{j}\left(\frac{N_{0}}{P}, K\right)}{1+\rho}\right)}$. It follows that as $L \rightarrow \infty$ for a fixed $K$ that the results in [1] still hold, which are summarized in the following proposition.

Proposition 1: Let $\rho^{*}$ and $m^{*}$ minimize (7) over $0 \leq \rho \leq 1$ and $m \geq 1$. If $L \rightarrow \infty$, then $\rho^{*}$ and $m^{*}$ satisfy $\rho^{*} \rightarrow 0$, $m^{*} \rightarrow \infty$, and $\frac{1}{\rho^{*}} m^{* 2} \log m^{*}=\Theta(L)$.

The proof of this follows from the same argument as Proposition 2 in [1] and so is omitted.

Proposition 1 shows the linear scaling of the total end-toend delay and the optimal scaling of $m^{*}$ and $\rho^{*}$ under a fixed reuse factor $K$. The coefficient of the highest order term in (7) is

$$
g\left(K, \frac{P}{N_{0}}\right):=\frac{K}{\sum_{j=1}^{K-1} \log \left(1+\frac{\gamma_{j}\left(\frac{N_{0}}{P}, K\right)}{1+\rho}\right)} .
$$


In [1], it is shown that the optimal $K$ minimizing the analogous term without redundant receptions is a bounded constant for all values of the SNR, $\frac{P}{N_{0}}$. Next, we consider whether this is still true with redundant receptions and compare the optimal reuse with that for the scheme without redundant receptions in [1]. For a given SNR, let $K_{2}$ be the value of $K$ minimizing $g\left(K, \frac{P}{N_{0}}\right)$ and let $K_{1}$ be the choice of $K$ that minimizes $K / \log \left(1+\frac{\gamma_{1}\left(\frac{N_{0}}{P}, K\right)}{1+\rho}\right)$, which is the highest order term for the scheme without redundant receptions. We want to compare $K_{1}$ and $K_{2}$ as a function of the SNR. To do this we consider several different SNR regimes.

We begin by studying the case where the SNR becomes asymptotically small.

Proposition 2: As $\frac{P}{N_{0}} \rightarrow 0, K_{2} \rightarrow 2$ and $K_{1} \rightarrow 2 .{ }^{4}$

Proof: Letting $\frac{P}{N_{0}} \rightarrow 0$ is equivalent to $\frac{N_{0}}{P} \rightarrow \infty$, in which case the noise component will be much larger than the interference part and so $\gamma_{j}\left(\frac{N_{0}}{P}, K\right) \rightarrow \frac{j^{-\alpha}}{\frac{N_{0}}{P}}$ and the limiting value of $g\left(K, \frac{P}{N_{0}}\right)$ is given by

$$
\begin{aligned}
\lim _{\frac{N_{0}}{P} \rightarrow \infty} g\left(K, \frac{P}{N_{0}}\right) & =\lim _{\frac{N_{0}}{P} \rightarrow \infty} \frac{K}{\sum_{j=1}^{K-1} \frac{j^{-\alpha}}{\frac{N_{0}}{P(1+\rho)}}} \\
& =\frac{K}{\sum_{j=1}^{K-1} j^{-\alpha}} \lim _{\frac{N_{0}}{P} \rightarrow \infty} \frac{\frac{N_{0}}{P}}{1+\rho} .
\end{aligned}
$$

Therefore, the $K$ minimizing $\frac{K}{\sum_{j=1}^{K-1} j^{-\alpha}}$ also minimizes $g\left(K, \frac{P}{N_{0}}\right)$ for small enough SNR. Since $\frac{K}{\sum_{j=1}^{K-1} j^{-\alpha}}$ is a monotonically increasing function of $K$ for all positive integers $K$, the smallest possible choice of $K$ yields the minimal value and so $K_{2} \rightarrow 2$. The same argument applies to $K_{1}$.

Next, we consider the case where $\frac{P}{N_{0}}$ is some finite non-zero constant.

Proposition 3: If $\frac{P}{N_{0}}$ is a finite non-zero constant, then $K_{2}$ is a finite constant and ignoring any integer constraints $K_{2} \geq$ $K_{1}$.

Proof: First, we will show that $K_{2}$ is finite. Consider

$$
\log \left(1+\frac{\gamma_{j}\left(\frac{N_{0}}{P}, K\right)}{1+\rho}\right)<\frac{\gamma_{j}\left(\frac{N_{0}}{P}, K\right)}{1+\rho}<\frac{j^{-\alpha}}{\frac{N_{0}}{P}(1+\rho)} .
$$

It then follows that

$$
g\left(K, \frac{P}{N_{0}}\right)>\frac{K}{\sum_{j=1}^{K-1}\left(\frac{j^{-\alpha}}{\frac{N_{0}}{P}(1+\rho)}\right)}>\frac{K}{\frac{1}{\frac{N_{0}}{P}(1+\rho)} \zeta(\alpha)},
$$

where $\zeta(\alpha)$ is the Riemann-Zeta function. Taking the limit as $K \rightarrow \infty$, it follows that

$$
\lim _{K \rightarrow \infty} g\left(K, \frac{P}{N_{0}}\right)>\lim _{K \rightarrow \infty} \frac{K}{\frac{1}{\frac{N_{0}}{P}(1+\rho)} \zeta(\alpha)}=\infty .
$$

Thus, the optimal $K_{2}$ has to be finite.

\footnotetext{
${ }^{4}$ We assume the minimal feasible $K$ is 2 due to the half-duplex constraint.
}

Next, we show that $K_{2} \geq K_{1}$. If we ignore any integer constraints on $K_{1}$ then this value should satisfy

$$
\left.\frac{\partial}{\partial K} \frac{K}{\log \left(1+\frac{\gamma_{1}\left(\frac{N_{0}}{P}, K\right)}{1+\rho}\right)}\right|_{K=\tilde{K}_{1}}=0 .
$$

If

$$
\left.\frac{\partial}{\partial K} g\left(K, \frac{N_{0}}{P}\right)\right|_{K=\tilde{K}_{1}} \leq 0
$$

then there must exist some $K$ larger than $\tilde{K}_{1}$ which yields a smaller value of $g\left(K, \frac{P}{N_{0}}\right)$. This implies that ignoring any integer constraints $K_{2} \geq K_{1}$.

To complete the proof, requires showing that (14) holds; the proof of this is sketched in the Appendix.

Finally, we consider the high SNR case, where the SNR $\frac{P}{N_{0}}$ goes to $\infty$.

Lemma 1: If $\frac{P}{N_{0}} \rightarrow \infty$, then no finite $K$ will minimize $g\left(K, \frac{P}{N_{0}}\right)$.

Proof: Let $f(x)$ denote function

$$
f(x)=\log \left(1+\frac{1}{1+\rho} \frac{x^{-\alpha}}{\sum_{i=1}^{\infty}\left((i+x)^{-\alpha}+(i-x)^{-\alpha}\right)}\right) .
$$

It can be easily shown that $f(x)$ is a convex decreasing function, with $\lim _{x=0} f(x)=\infty$, and $f(1)=0$.

The $K$ minimizing $g(K, \infty)$ is the same as the $K$ maximizing

$$
\frac{1}{K} \sum_{j=1}^{K-1} \log \left(1+\frac{\gamma_{j}\left(\frac{N_{0}}{P}, K\right)}{1+\rho}\right)=\frac{1}{K} \sum_{j=1}^{K-1} f\left(\frac{j}{K}\right)
$$

which can be seen to be a lower bound on the integral of this function $f(x)$. As $K$ becomes large, the resulting bound will approach the Riemann integral of $f(x)$ and thus the result follows.

Proposition 4: As $\frac{P}{N_{0}} \rightarrow \infty$, then $K_{2} \rightarrow \infty$.

Proof: Applying Lemma 1, the result follows.

This result implies that for large enough values of $P / N_{0}$, we'd prefer to have at most one node transmitting at a time and all nodes would participate in the transmission. Note that the numerator of $g$ in (8) reflects the increase in end-to-end delay due to a loss in pipe-lining with larger choices of $K$. The denominator of $g$ reflects the decrease in delay due to an increase in the "rate per hop" due to lower interference and a larger cooperative group of users. This result shows that for high SNRs the later effect dominates.

\section{B. Concatenated Decoding Scheme}

In [1], a concatenated coding scheme was also considered in which an end-to-end outer code is used to correct missing packets that do not arrive at the destination. For the scheme without redundant reception, it was shown that the total end-toend delay still grows linearly with $L$, but that the concatenated coding scheme has smaller end-to-end delay due to slower growth in the second highest order terms. 
We can consider combining our scheme with redundant receptions with such a concatenated code. Using the same formulation in [1] and applying the similar argument as in Section III-A, the scheme with or without the redundant reception again have the same order of growth and differ only in the highest order coefficient. In particular it can be shown that all the results given in Section III-A still hold for such a scheme. We omit the details due to space considerations.

\section{NumericAl Results}

In this section, we present some numerical results for nonasymptotic systems. First in Fig. 2 we show the growth order of the minimal delays using for the scheme with redundant reception both with and without concatenated coding. We also plot the first order terms for each scheme. It is clear that the highest order term dominates the total delay. We also show the behavior of the optimal $K$ for different messages sizes $L$. Figure 3 shows the optimal $\rho$ and $m$ as a function of $L$. The results shown in these two figures are consistent with our analysis. In all of these figures, $\alpha=3, \eta=0.001, N_{0} / P=1$, $h=10$, and $H=10$.
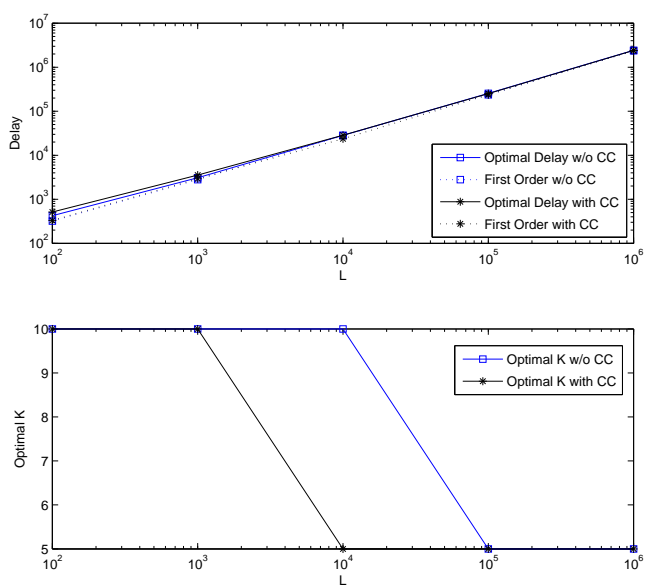

Fig. 2. Minimum delays and optimal $K$ 's with redundant receptions.

Next we compare the delay and reuse factors between using redundant receptions and not using them as in [1]. Figure 4 shows the delay gain of the scheme with redundant reception over the scheme without it. The ratio of the optimal delay with redundant receptions to that without redundant receptions is shown as a function of SNR for different choices of the path loss constant $\alpha$. As expected, the ratio decreases with SNR. For a given SNR, the ratio increases with $\alpha$.

Figure 5 and Figure 6 show the optimal reuse factor $K$ versus the SNR for the scheme with and without redundant receptions, respectively. These two figures show that as predicted, the optimal $K$ for the scheme with redundant reception increases without bound while the optimal $K$ for the other scheme converges to a bounded constant.
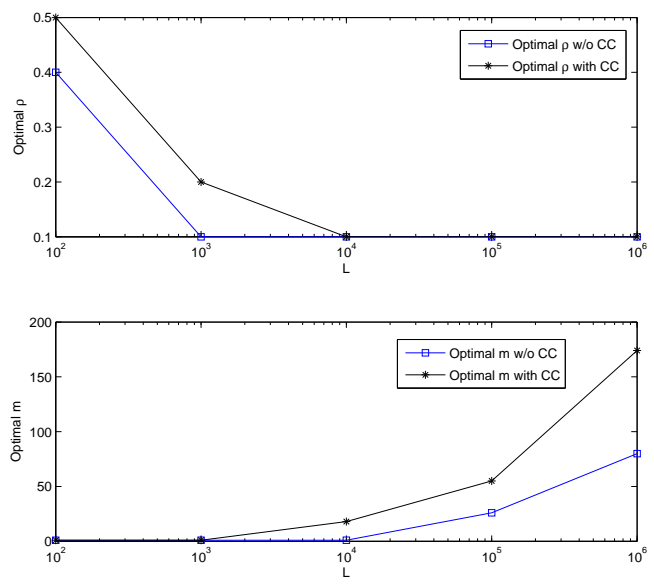

Fig. 3. Optimal $\rho$ 's and $m$ 's.

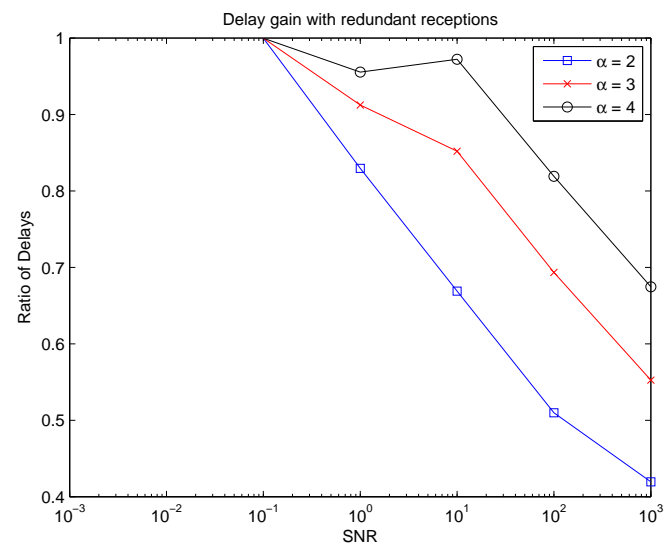

Fig. 4. The ratio of the optimal delay with redundant receptions to that without as a function of SNR.

\section{CONCLUding REMARKS}

In this paper, we considered a linear ad hoc network in which nodes combine multiple copies of a message received with different SINRs. By jointly decoding, the performance in terms of delay for a given reliability is boosted compared to the schemes studied in [1] in which decoding was based only on the transmission of the nearest node.

We also considered the delay performance under different SNR settings. In the low SNR regime, the noise dominates, and using redundant receptions gives little improvement relative to the schemes in [1]. In this regime, both schemes have a similar optimal reuse factor $K$. On the other hand, in the high SNR regime, the scheme with redundant receptions has a better performance gain and much larger optimal $K$ than the previous scheme. In the limiting case, where the SNR goes to $\infty$, the analysis suggests that the reuse factor of the redundant reception scheme will become arbitrarily large. This implies no reuse, which is quite different from the scheme without redundant receptions. 


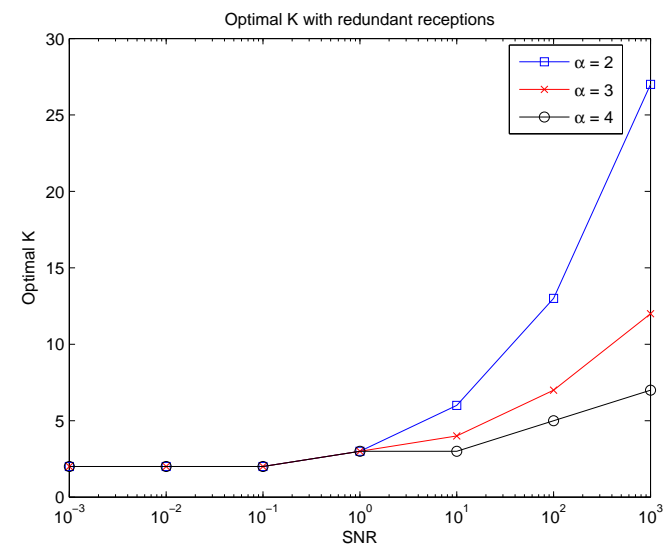

Fig. 5. Optimal $K$ vs. SNR with redundant receptions.

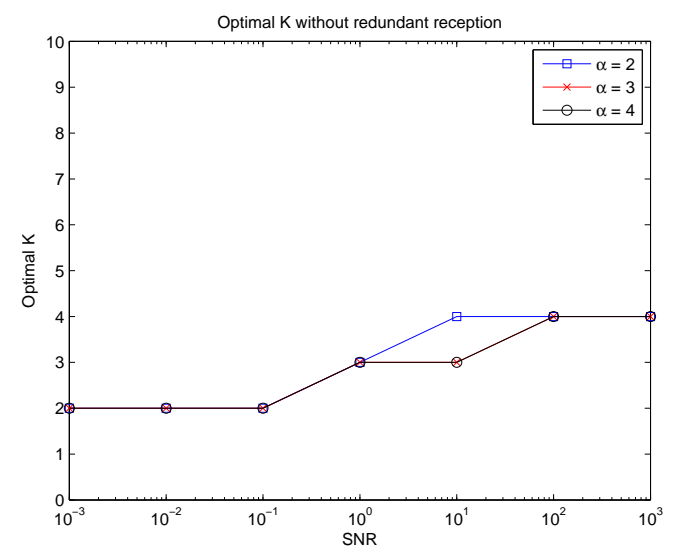

Fig. 6. Optimal $K$ vs. SNR without redundant receptions.

\section{REFERENCES}

[1] N. Wen and R. Berry, "Reliability constrained packet-sizing for linear multi-hop wireless networks," in Information Theory, 2008. ISIT 2008. IEEE International Symposium on, July 2008, pp. 16-20.

[2] W. Zhang and U. Mitra, "A delay-reliability analysis for multihop underwater acoustic communication," in WuWNet '07: Proceedings of the second workshop on Underwater networks. New York, NY, USA: ACM, 2007, pp. 57-64.

[3] — "Multihopping strategies: An error-exponent comparison," in IEEE International Symposium on Information Theory (ISIT), Nice, France, June 2007.

[4] O. Oyman, "Reliability bounds for delay-constrained multi-hop networks," in 44th Annual Allerton Conference, Illinois, Sept. 2006.

[5] M. Sikora, J. N. Laneman, M. Haenggi, D. J. Costello, and T. Fuja, "On the Optimum Number of Hops in Linear Ad Hoc Networks," in IEEE Information Theory Workshop (ITW'04), San Antonio, TX, Oct 2004.

[6] G. Kramer, M. Gastpar, and P. Gupta, "Cooperative strategies and capacity theorems for relay networks," Information Theory, IEEE Transactions on, vol. 51, no. 9, pp. 3037-3063, Sept. 2005.

[7] R. Liu, P. Spasojevic, and E. Soljanin, "Incremental multi-hop based on good punctured codes and its reliable hop rate," in Proceedings. IEEE Wireless Communications and Networking Conference 2004 (WCNC 2004), Atlanta, Georgia, March 2004.

[8] R. G. Gallager, Information Theory and Reliable Communication. New York, NY, USA: John Wiley \& Sons, Inc., 1968.

\section{APPENDIX}

It can be shown that

$$
\begin{aligned}
& \left.\left.\left.\frac{\partial}{\partial K} g\left(K, \frac{P}{N_{0}}\right)\right|_{K=K_{1}}\right|_{=} \frac{1}{\left(\sum_{j=1}^{K-1} \log \left(1+\frac{\gamma_{j}\left(\frac{N_{0}}{P}, K\right)}{1+\rho}\right)\right)^{2}}\right|_{K=K_{1}} \times \\
& {\left[\sum_{j=2}^{K-1}\left(\log \left(1+\frac{\gamma_{j}\left(\frac{N_{0}}{P}, K\right)}{1+\rho}\right)\right)^{2} \times\right.} \\
& \left.\frac{\partial}{\partial K}\left(\frac{K}{\log \left(1+\frac{\gamma_{j}\left(\frac{N_{0}}{P}, K\right)}{1+\rho}\right)}\right)\right|_{K=K_{1}} .
\end{aligned}
$$

Notice that for any $j \geq 2, \leq K-1,{ }^{5}$

$$
0=\gamma_{1}\left(\frac{N_{0}}{P}, 0\right)<\gamma_{j}\left(\frac{N_{0}}{P}, K\right)<\gamma_{1}\left(\frac{N_{0}}{P}, K\right) .
$$

Hence, for any $K$, there must exist some $K^{\prime}<K$ such that $\gamma_{j}\left(\frac{N_{0}}{P}, K\right)=\gamma_{1}\left(\frac{N_{0}}{P}, K^{\prime}\right)$. Thus

$$
\begin{aligned}
& \left.\left.\frac{\partial}{\partial K}\left(\frac{K}{\log \left(1+\frac{\gamma_{j}\left(\frac{N_{0}}{P}, K\right)}{1+\rho}\right)}\right)\right|_{K=K_{1}}\right) \\
= & \left\{\left[\frac{K}{K^{\prime}} \frac{\partial}{\partial K^{\prime}}\left(\frac{K^{\prime}}{\log \left(1+\frac{\gamma_{1}\left(\frac{N_{0}}{P}, K^{\prime}\right)}{1+\rho}\right)}\right)+\right.\right. \\
& \left.\left.\left(\frac{\left.K^{\prime}\right)}{\log \left(1+\frac{\gamma_{1}\left(\frac{N_{0}}{P}, K^{\prime}\right)}{1+\rho}\right)}\right) \frac{\partial}{\partial K^{\prime}} \frac{K}{K^{\prime}}\right] \frac{\partial K^{\prime}}{\partial K}\right\}\left.\left.\right|_{K=K_{1}}\right|_{(19)}
\end{aligned}
$$

When $K=K_{1}$, then for any $K^{\prime}<K_{1}$, $\frac{\partial}{\partial K^{\prime}}\left(\frac{K^{\prime}}{\log \left(1+\frac{\gamma_{1}\left(\frac{N_{0}}{P}, K^{\prime}\right)}{1+\rho}\right)}\right)<0$.
$\quad$ Clearly, $\frac{K}{K^{\prime}}>0, \frac{K^{\prime}}{\log \left(1+\frac{\gamma_{1}\left(\frac{N_{0}}{P}, K^{\prime}\right)}{1+\rho}\right)}>0$, and $\frac{\partial K^{\prime}}{\partial K}>0$. Now consider $\frac{\partial}{\partial K^{\prime}} \frac{K}{K^{\prime}}$ and note that $\gamma_{j}\left(\frac{N_{0}}{P}, K\right)$ is

$$
\frac{1}{\frac{N_{0}}{P} j^{\alpha}+\sum_{i=1}^{\infty}\left[(i(K / j)+1)^{-\alpha}+(i(K / j)-1)^{-\alpha}\right]} \text {. }
$$

If $\frac{N_{0}}{P}=0$, then $K^{\prime}=K / j$. Since we consider $\frac{N_{0}}{P}>0$, $K^{\prime}>K / j$. However, if $K \rightarrow 0$, then interference dominates and $K^{\prime} \rightarrow K / j$. As $K$ grows, $K^{\prime}$ moves away from $K / j$ and $K^{\prime}>K / j$. Thus, $\frac{\partial}{\partial K^{\prime}} \frac{K}{K^{\prime}}<0$.

Combining the above observations it follows that (19) is less than 0 and the final result follows.

${ }^{5} \mathrm{Here}$, we assume that $\gamma_{1}\left(\frac{N_{0}}{P}, K\right)$ is well defined around 0 , and continuous in $K$. 